\title{
Electrode Polarisation, dielectrophoresis and electrorotation
}

\author{
Nicolas G Green \\ AC Electrokinetics and Electrohydrodynamics Group \\ Department of Electronics and Electrical Engineering, University of Glasgow, \\ Oakfield Avenue, Glasgow G12 8LT, Scotland, UK \\ n.green@elec.gla.ac.uk
}

\begin{abstract}
Dielectrophoresis (DEP) and Electrorotation (ROT) are used for the characterisation, identification, manipulation and separation of a wide range of bioparticles, such as cells, bacteria and viruses. The particles are suspended in aqueous solutions of ions and the electric fields are generated by arrays of microelectrodes by the application of AC potential signals. When a potential is applied across an electrolyte using electrodes, an electrical double layer forms in the fluid above the electrode surface, screening the potential from the bulk and reducing the electric field in the bulk. This paper discusses a linear solution of electrode polarisation in microelectrode geometries and the frequency dependent effect on dielectrophoresis and electrorotation. The movement of bioparticles, such as cells, under these conditions is then discussed as a function of frequency
\end{abstract}

\section{Introduction}

Dielectrophoresis (DEP) is the movement of polarisable particles resulting from the interaction of a non-uniform electric field and the dipole induced in the particles $[1,2]$. Electrorotation (ROT) is the rotation resulting from the interaction of a rotating electric field and the particle [1]. Collectively referred to as AC Electrokinetics, these techniques have been used for the characterisation, identification, manipulation and separation of a wide range of bioparticles, such as cells, bacteria and viruses. In practice, the particles are suspended in aqueous solutions of ions (electrolytes), with a range of conductivities. The electric fields are generated by arrays of microelectrodes from the application of AC potential signals [2].

When a potential is applied across an electrolyte using electrodes, an electrical double layer forms in the fluid above the electrode surface. In many cases, the charging of the layer can be considered to be analogous to the charging and polarising of a capacitor, and the mechanism is often referred to as electrode polarisation. The double layer consists of the counterionic species to the applied potential, attracted to the surface by the potential and repelled by diffusion. At low frequencies, the charging of the double layer screens the potential from the bulk, reducing the electric field experienced by the particle in the bulk [3].

For microelectrode structures and AC potential signals, the charging of the electrical double layer is a complicated, frequency and geometry dependent problem. The reduction in the electric field strength leads to a reduction in the dielectrophoretic force. In addition, the non-uniform field interacts with the double layer to produce a tangential flow, referred to as 
AC electroosmosis [4]. This paper discusses electrode polarisation in microelectrode geometries typically used in AC electrokinetic experiments and the frequency dependent effect on dielectrophoresis and electrorotation. The model is based on a linear model of the double layer, determined from experimental measurements of the double layer impedance. The movement of a variety of bioparticles under these conditions is also discussed

\section{Background, theory and numerical simulation}

\subsection{Dielectrophoresis}

Dielectrophoresis is the movement of a particle caused by the interaction of a non-uniform electric field and the dipole moment it induces in the particle. The time-averaged dielectrophoretic force $\left\langle\mathbf{F}_{D E P}\right\rangle$ is generally written as [1,2]

$$
\left\langle\mathbf{F}_{D E P}\right\rangle=\frac{1}{4} v \operatorname{Re}[\tilde{\alpha}] \nabla|\mathbf{E}|^{2}
$$

where $v$ is the volume of the particle, $\tilde{\alpha}$ is the complex, frequency-dependent effective polarisability of the particle, $\operatorname{Re}[\ldots]$ indicates Real part of and $\mathbf{E}$ is the electric field phasor. This expression is valid if the electric field phasor is only real. If the electric field phasor is complex, the dielectrophoretic force is given by

$$
\left\langle\mathbf{F}_{D E P}\right\rangle=\frac{1}{4} v \operatorname{Re}[\tilde{\alpha}] \nabla\|\mathbf{E}\|^{2}-\frac{1}{2} v \operatorname{Im}[\tilde{\alpha}] \nabla \times(\operatorname{Re}[\mathbf{E}] \times \operatorname{Im}[\mathbf{E}])
$$

where $\operatorname{Im}[\ldots]$ indicates the imaginary part of. This is the expression required for travelling wave dielectrophoresis, with the travelling wave force given by the second term on the right hand side of equation (2).

If there is a spatial variation in the phase of the field: if the field phasor is complex, the travelling wave part of the dielectrophoretic force is non-zero.

\subsection{Electrorotation}

Electrorotation is the rotation of a particle caused by the interaction of a rotating electric field and the induced dipole moment. The time-averaged electrorotational torque is

$$
\left\langle\mathbf{G}_{R O T}\right\rangle=-v \operatorname{Im}[\tilde{\alpha}](\operatorname{Re}[\mathbf{E}] \times \operatorname{Im}[\mathbf{E}])
$$

This torque is also non-zero in a complex field.

\subsection{Frequency dependent polarisability}

The frequency dependent polarisability $\tilde{\alpha}$ in equations (1), (2) and (3) is, for a solid spherical particle, given by

$$
\tilde{\alpha}=3 \varepsilon_{m}\left(\frac{\tilde{\varepsilon}_{p}-\tilde{\varepsilon}_{m}}{\tilde{\varepsilon_{p}}+2 \tilde{\varepsilon}_{m}}\right)
$$


(a)

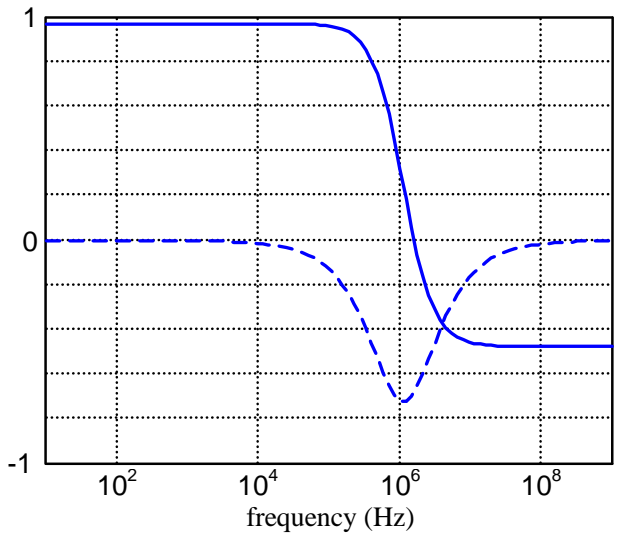

(b)

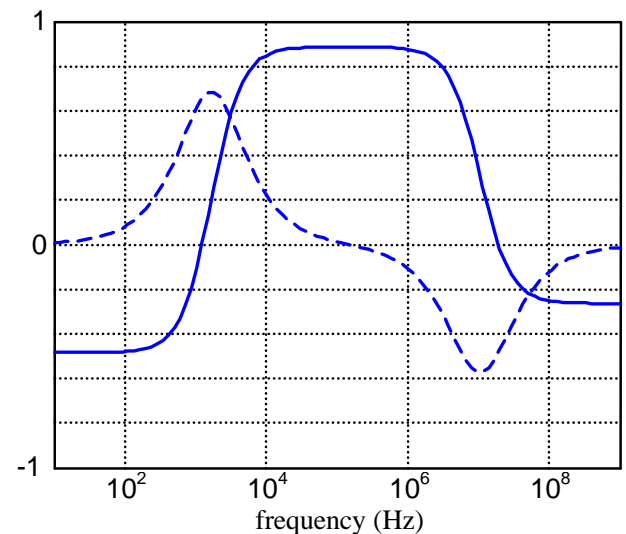

Figure 1 Plots of the real (solid line) and imaginary (dashed line) of the Clausius-Mossotti factor for (a) a solid spherical particle and (b) the two shell model for a biological cell with membrane, cytoplasm and nucleus.

where $\varepsilon_{m}$ is the permittivity of the suspending fluid and $\tilde{\varepsilon}_{m}$ and $\tilde{\varepsilon}_{p}$ is the complex permittivities of the fluid and particle respectively. A general complex permittivity is given by $\tilde{\varepsilon}=\varepsilon-i \sigma / \omega$ where $\varepsilon$ is the permittivity, $\sigma$ is the conductivity, $\omega$ is the frequency of the applied AC field and $i$ is the imaginary unit.

The term in brackets in equation (4) is referred to as the Clausius-Mossotti factor and describes the frequency dependence of the polarisability. Figure (1) shows plots of the real and imaginary parts of the Clausius-Mossotti factor for a solid particle and a biological cell, modelled using the shell model $[1,2]$.

\section{Numerical simulation of the electrical potential}

\subsection{Polarisation of the double layer: Electrode Polarisation}

In this paper, we will assume that the polarisation of the double layer is linear and that it can be represented by a constant capacitance across the electrode surface. This is an approximation that will not be valid close to the electrode edge where the field is strongest. The double layer also consists of two distinct layers, the diffuse layer consisting of a cloud of counterions which have close to bulk diffusion properties and the Stern layer which consists of a layer of packed hydrated ions and possibly bare adsorbed ions [3]. One possible method for avoiding potentially complicated numerical simulation of the polarisation process is to use the experimentally measured impedance of the double layer.

\subsection{Simulation of potential and electric field}

The electrical potential in the bulk, $\phi$, is then a solution of Laplace's equation with the boundary condition at the electrode surface given by

$$
\sigma \frac{\partial \phi}{\partial y}=\frac{1}{Z_{D L}}\left(\phi-V_{o}\right)
$$

where $Z_{D L}$ is the measured impedance of the double layer and $V_{o}$ is the potential applied to the electrode. The complex potential $\phi=\phi_{R}+i \phi_{I}$, is solved in the problem space shown in figure (2), with the symmetry boundary conditions as shown and the above expression for 
the boundary condition on the electrode. The electrodes are assumed to infinitely long, so that the problem can be assumed to be two-dimensional. The symmetrical nature of the electrodes means that has to be solved is half of one electrode and half of the adjacent gap.

The potential was solved using FlexPDE $^{\circledR}$, a commercially available Finite Element Partial Differential Equation solver, for a conductivity of $2 \times 10^{-3} \mathrm{Sm}^{-1}$ and electrode width of $10 \mu \mathrm{m}$. The problem was posed as the simultaneous solution of the Laplace equation for the real and imaginary parts of the potential, linked by the boundary condition, given by equation (5) on the electrode. The resulting potential at the electrode surface is shown in Figure (3). The real part of the potential decreases with frequency, but importantly, does not decrease in the same manner across the electrode

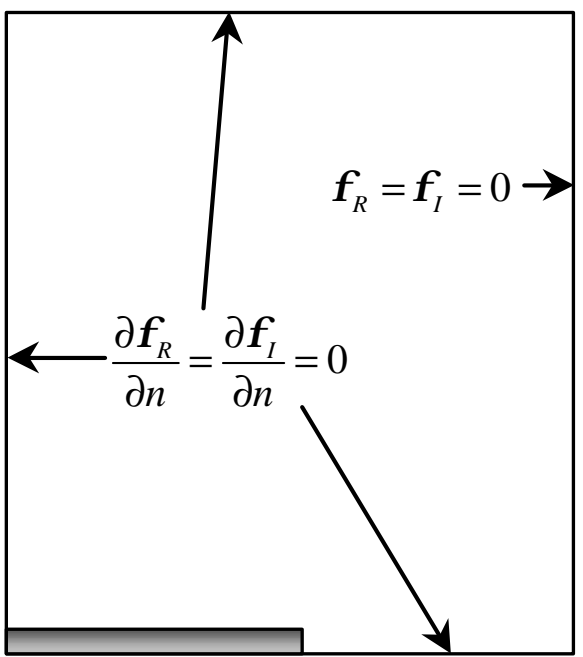

Figure 2 The problem space for the complex potential $\phi$ and the boundary conditions.
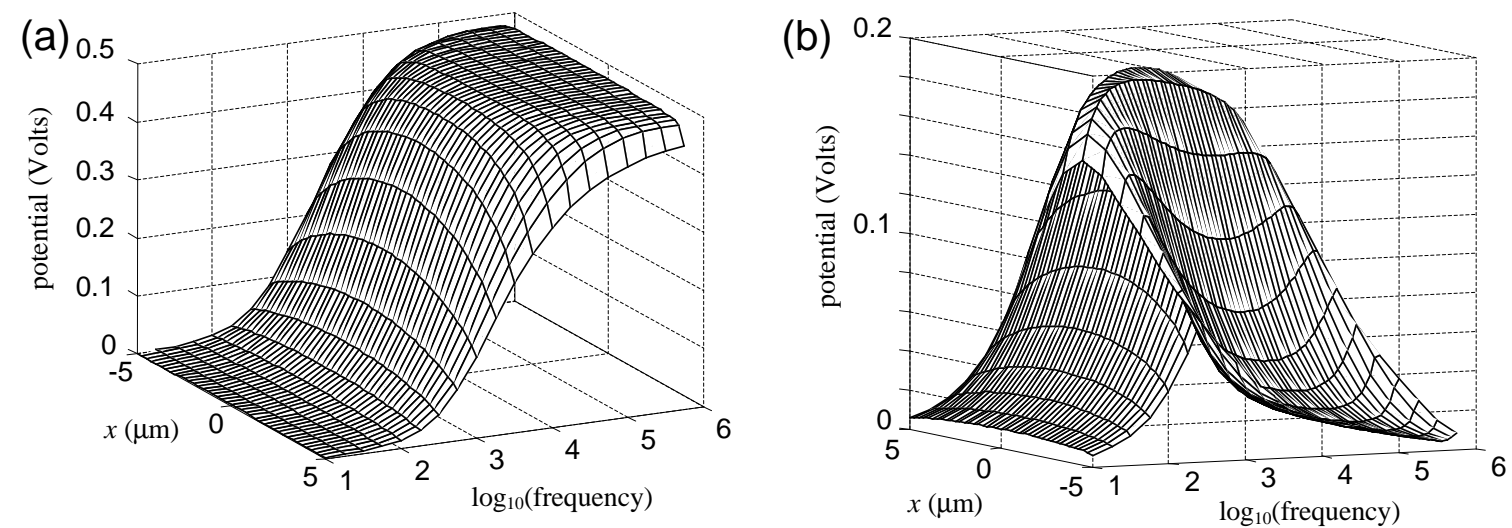

Figure 3 Plot of the real (a) and imaginary (b) parts of the complex potential at the surface of the electrode plotted as a function of frequency and distance from the centre.

(a)

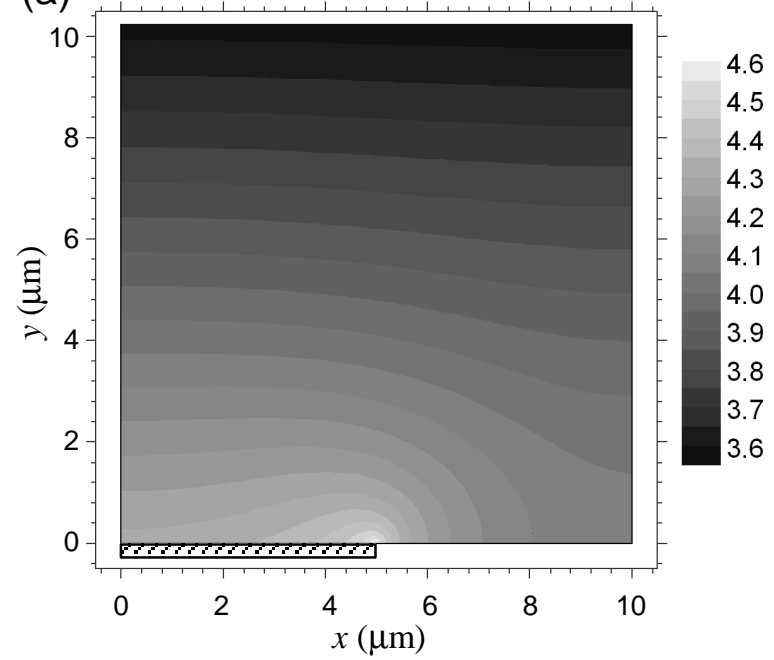

(b)

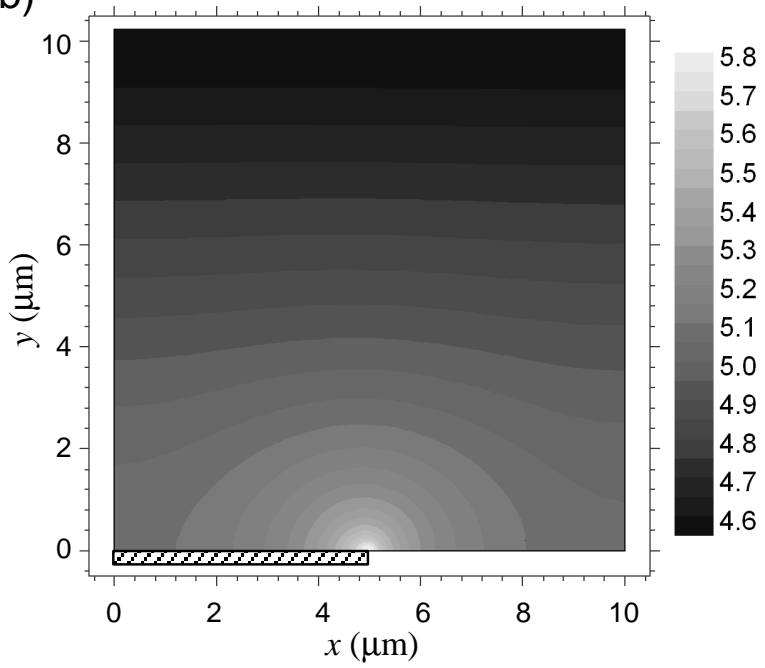

Figure 4 Plot of the magnitude of the electric field in the problem space at (a) $100 \mathrm{~Hz}$ and (b) $100 \mathrm{kHz}$. The greyscale for the plots is $\log _{10}$ (magnitude). 
Surface. The imaginary part is maximum around $1 \mathrm{kHz}$ for this conductivity. The magnitude of the resulting electric field is shown in Figure (4) for two frequencies, $100 \mathrm{~Hz}$ (a) and $100 \mathrm{kHz}(\mathrm{b})$. At high frequencies, the electric field magnitude is symmetrical about the edge of the electrode at $5 \mu \mathrm{m}$. At low frequencies, the electric field magnitude is no longer symmetrical. The effect of this change in field distribution can be seen in Figure (5), where at high frequencies the DEP force (figure 5(a) and the first term in equation (2)) is symmetrical about the electrode edge, but at low frequencies (figure 5(b)) the DEP force direction is no longer symmetrical. Note that the magnitude is much smaller (c.f. figure (7)).

In addition to this alteration to the DEP force, the twDEP force is non-zero (second term in equation (2)). Figure (6) shows the twDEP force component direction for the same two frequencies, the magnitude of which is highest around $1 \mathrm{kHz}$. This will not affect the solid sphere (figure $1(\mathrm{a})$ ) since $\operatorname{Im}[\tilde{\alpha}]=0$ but will affect the cell since the $\operatorname{Im}[\tilde{\alpha}] \neq 0$.

However, this additional force is small compared to the DEP force component. What is

(a)

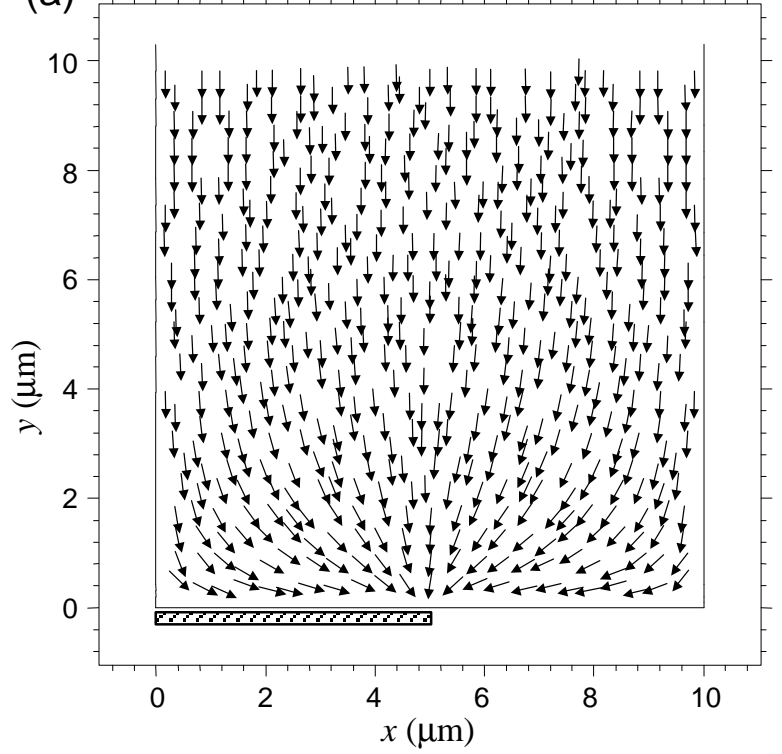

(b)

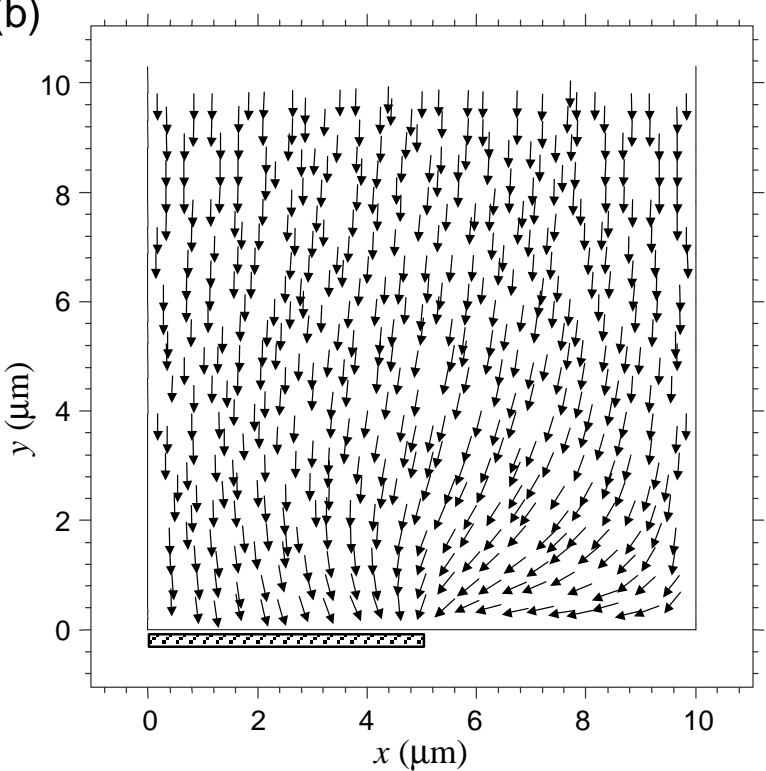

Figure 5 Plot of direction of $\nabla\|\mathbf{E}\|^{2}$ at (a) $100 \mathrm{kHz}$ and (b) $100 \mathrm{~Hz}$.

(a)

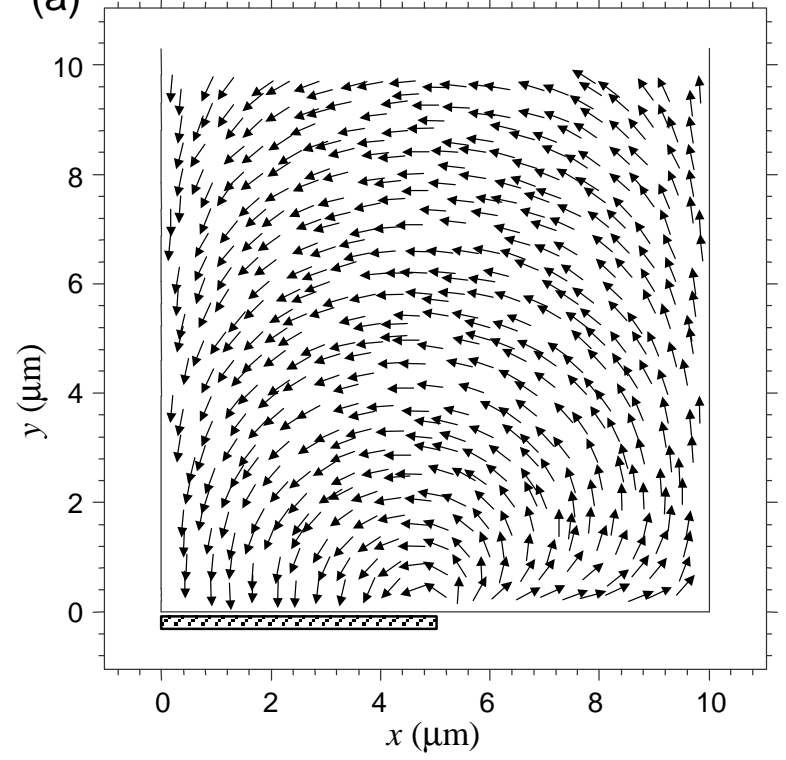

(b)

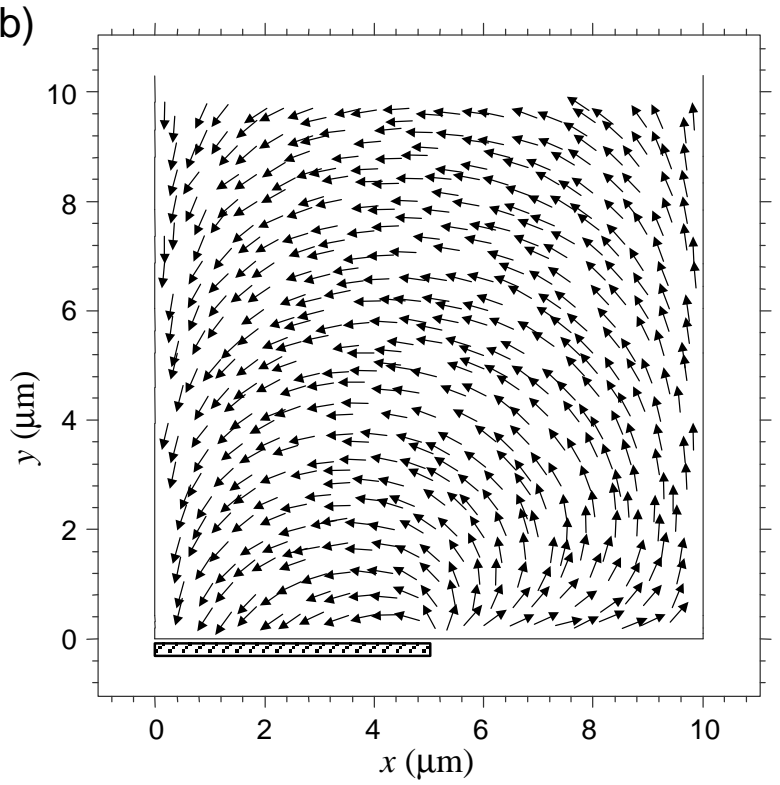

Figure 6 Plot of direction of $\nabla \times(\operatorname{Re}[\mathbf{E}] \times \operatorname{Im}[\mathbf{E}])$ at (a) $100 \mathrm{kHz}$ and (b) $100 \mathrm{~Hz}$. 

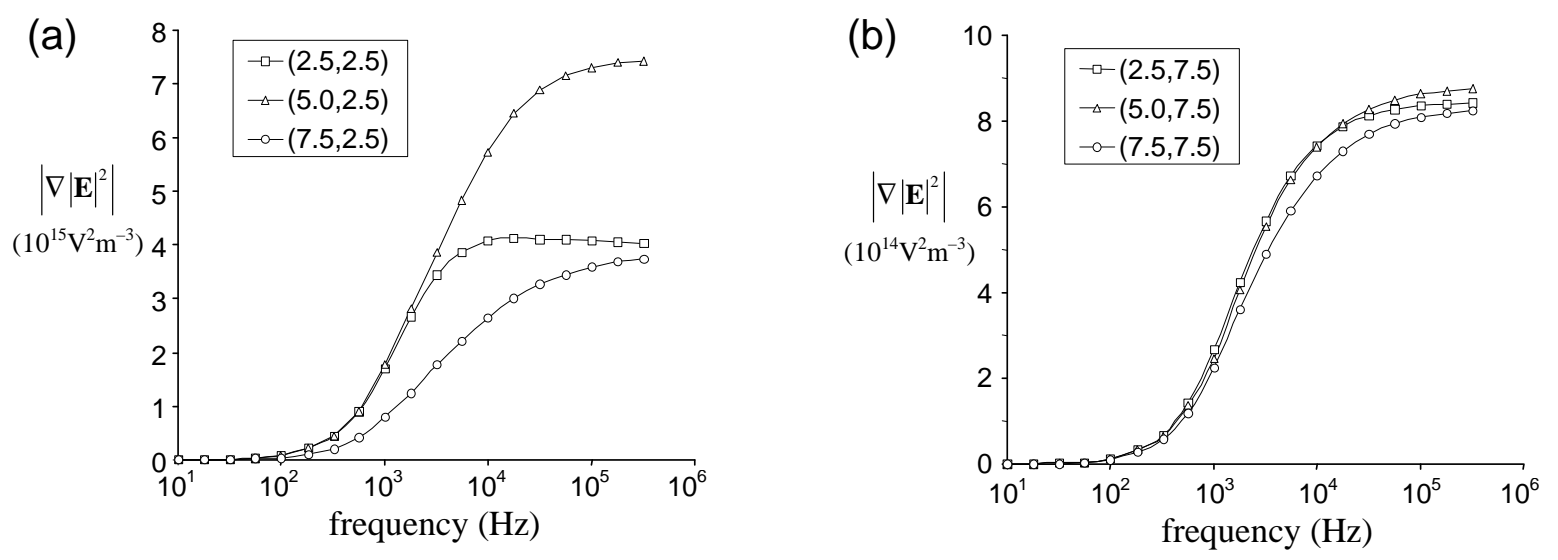

Figure 7 Plot of magnitude of the DEP force for three positions (indicated by the legend) along $y$ $=2.5 \mu \mathrm{m}(\mathrm{a})$ and $y=7.5 \mu \mathrm{m}(\mathrm{b})$, showing the variation in the frequency dependency.

significant is the fact that at distances less than the typical size of the electrodes, the frequency dependence of the DEP force is spatially dependent as shown in Figure (7). In addition, there is a substantial electrorotational torque acting on particles close to the electrode edge, as shown by figure (8).

\section{Conclusion}

The effects of electrode polarisation in planar microelectrodes is not as simple a problem to solve as multiplying the DEP force by a fixed frequency dependent factor. The non-uniform nature of the electrode polarisation leads to a spatially

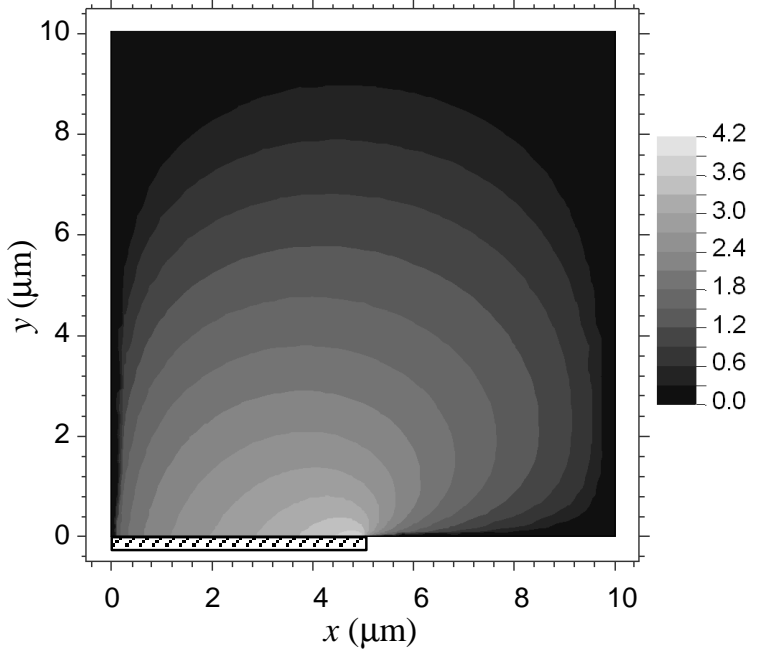

Figure 8 Plot of the rotation rate of a typically particle due to the electrorotational torque. The greyscale is $\log _{10}\left(\mathrm{rad} \mathrm{s}^{-1}\right)$. dependent frequency variation as well as, potentially, travelling wave effects and a strong electrototational torques close to the electrode edge. These results were based on a linear model of the double layer and a better picture of experimental results would require more accurate double layer models.

\section{Acknowledgements}

The author is a Royal Academy of Engineering Post-doctoral Research Fellow at the University of Glasgow. The author would also like to thank Professor Hywel Morgan, Professor Antonio Castellanos, Dr Antonio Ramos and Dr Antonio González for valuable discussions.

\section{References}

[1] Jones T.B. Electromechanics of Particles, Cambridge Univ. Press, Cambridge (1995)

[2] Morgan H. and Green N.G. AC Electrokinetics: Colloids and Nanoparticles, Research Studies Press, Baldock, UK (2003)

[3] Lyklema J. Fundamentals of Interface and Colloid Science Acad. Press Ltd, London, (1995).

[4] Green N.G., Ramos A., González A., Morgan H. and Castellanos A. Fluid flow induced by nonuniform AC electric fields in electrolytes on microelectrodes III: Observation of streamlines and numerical simulation Phys RevE $\mathbf{6 6}$ art no. 026305 (2002) 